\title{
Le picaresque dans le Manuscrit trouvé à Saragosse : narration et mensonge
}

\section{Lena Seauve}

\section{(2) OpenEdition}

\section{Journals}

Édition électronique

URL : http://journals.openedition.org/edl/465

DOI : $10.4000 /$ edl. 465

ISSN : 2296-5084

Éditeur

Université de Lausanne

\section{Édition imprimée}

Date de publication : 15 décembre 2012

Pagination : 165-182

ISBN : 978-2-940331-29-1

ISSN : 0014-2026

\section{Référence électronique}

Lena Seauve, "Le picaresque dans le Manuscrit trouvé à Saragosse : narration et mensonge ", Études de lettres [En ligne], 4 | 2012, mis en ligne le 15 décembre 2015, consulté le 19 décembre 2020. URL: http://journals.openedition.org/edl/465 ; DOI : https://doi.org/10.4000/edl.465 


\section{LE PICARESQUE DANS \\ LE MANUSCRIT TROUVÉ À SARAGOSSE: NARRATION ET MENSONGE}

L'article aborde la question de savoir de quelle manière l'influence du genre picaresque se manifeste dans le Manuscrit trouvé à Saragosse (versions de 1804 et de 1810) de Jean Potocki. La présence du picaresque dans le Manuscrit peut être, c'est l'hypothèse de l'étude, expliquée par la qualité principalement intertextuelle du roman. A partir de l'exemple du récit du bohémien Avadoro, le personnage et le narrateur picaresque sont l'objet d'une analyse qui se penche surtout sur la problématique de la narration et du mensonge dans le roman.

\section{Pourquoi le picaresque?}

La critique a souvent - et à juste titre - rapproché le roman de Jean Potocki de différents grands textes fondateurs de la littérature européenne moderne comme les Mille et une nuits ou le Décaméron de Boccace. L'établissement de ces parallèles s'explique notamment par une technique utilisée dans le Manuscrit que François Rosset désigne par la notion de «réemploi » ${ }^{1}$. Cette pratique, que l'on pourrait compter parmi les pratiques intertextuelles, consiste en la réutilisation de certaines composantes d'œuvres et de genres antérieurs dans un nouveau texte. Ces éléments réutilisés peuvent comprendre des unités lexicales, des séquences narratives ou même des dispositifs narratifs élaborés ${ }^{2}$.

I. F. Rosset, «Le langage du fantastique», p. 203.

2. Ibid., p. 204. 
Cela signifie qu'un roman comme le Manuscrit se réapproprie des éléments typiques de certains genres littéraires, sans que l'on puisse dire que le roman dans son ensemble appartienne aux genres évoqués. Au contraire, l'utilisation de ces éléments génériques se manifeste souvent sous la forme d'un changement décisif de ton. Tout en se référant clairement à un genre littéraire existant, le Manuscrit en change ainsi l'orientation habituelle. Si, par exemple, le Manuscrit reproduit des constellations caractéristiques du roman gothique anglais, il en laisse de côté un élément essentiel: la peur des personnages qui sont confrontés à des événements inexplicables. L'effet principal que le roman gothique pourrait ainsi avoir sur son lecteur est donc fondamentalement changé dans la manière potockienne de se servir des éléments typiques du genre.

Heinrich Julius von Klaproth, qui avait accompagné Potocki lors de son voyage en Chine, avait déjà nommé, parmi les textes de référence du Manuscrit, L'Histoire de Gil Blas de Santillane de Lesage ${ }^{3}$, roman écrit dans la plus pure tradition picaresque espagnole. Voilà la première indication pour un rapprochement entre le Manuscrit et le roman picaresque. Le choix de l'Espagne - et notamment de la Sierra Morena, où se déroule aussi une partie du Don Quichotte, roman qui contient, comme le Manuscrit, des épisodes picaresques - comme lieu d'action peut être considéré comme un deuxième indice. Plusieurs personnages figurant dans le roman, comme le chef bohémien ou le bandit Zoto, présentent à l'évidence des traits de caractère typiquement picaresques: ils mentent, ils atteignent leur but en trompant leur prochain et ils changent d'identité plusieurs fois. Contrairement à ce que l'on pourrait attendre au vu d'une parenté si évidente, le rapport entre la tradition picaresque et le Manuscrit n'a fait l'objet jusqu'à ce jour que d'une seule étude, celle de Julien Hervier, qui date de $1985^{4}$. Cette analyse, qui se réfère à l'édition incomplète de Caillois, nous servira de point de départ pour développer quelques idées sur les rapports entre la tradition picaresque et le mode de narration dans le Manuscrit. Tandis que l'analyse d'Hervier choisit une approche principalement sociologique, la présente étude se penchera plutôt sur les aspects narratologiques du picaresque dans le Manuscrit. Hervier part du principe que Potocki, en tant que membre de la haute

3. Cf. M. Zurowski, «Le Manuscrit trouvé à Saragosse et la technique romanesque du XVIII" siècle», p. 106.

4. J. Hervier, "Jean Potocki ou les métamorphoses du picaresque». 
noblesse polonaise, reflète dans son roman la position spécifique de la noblesse européenne par rapport à une bourgeoisie ascendante; dès lors, son roman traiterait, par le détour de la fiction picaresque, de l'affaiblissement des valeurs féodales par rapport aux idées progressistes des Lumières. Selon Hervier, le Manuscrit serait un traité de morale invitant l'aristocratie à se souvenir de l'importance de ses valeurs anciennes:

[...] le cadre picaresque, avec son opposition traditionnelle entre l'aristocrate et le picaro, se révèle merveilleusement conforme à son dessein. Mais tandis que l'antihonneur du picaro démasquait l'honneur factice de l'aristocrate (Lazarillo) ou l'humiliait devant Dieu au sein de l'universelle bassesse humaine (Guzman), le contre-honneur du bandit potockien n'est qu'un des avatars de l'honneur ancien. La morale féodale du point d'honneur et du courage, raillée sous les formes ingénues qu'elle revêt chez Alphonse ou lorsqu'elle prétend constituer à elle seule toute une éthique, est restaurée sur d'autres bases 5 .

Cette approche sociologique, voire biographique, semble cependant difficilement justifiable face à un roman dont les personnages et les constellations sociales sont dénués de tout réalisme, historique ou non. De même que Potocki ne semble pas vraiment s'intéresser à ce qu'on pourrait, de manière anachronique, appeler la psychologie de ses personnages, le Manuscrit ne représente pas, comme on pourrait le croire, un monde existant - et pas davantage un monde ayant existé à un moment historique précis. Le roman ne parle pas du réel, il se réfère essentiellement à des œuvres de fiction. Les lieux, les sociétés et les personnages qui sont décrits sont, on le verra plus loin, des configurations fictives; il s'agit donc avant tout d'un phénomène d'intertextualité et non pas de rapport au réel.

\section{Définition du picaresque - caractéristiques de la narration picaresque}

Un des premiers problèmes qui se pose si l'on veut parler du roman picaresque, c'est d'abord le fait que traditionnellement, l'histoire littéraire limite ce genre à l'époque située entre 1550 et 1650 . Le roman picaresque

5. Ibid. 
serait, selon cette délimitation étroite, un phénomène principalement espagnol, qui comprend surtout les textes fondateurs du genre, comme le Lazarillo de Tormes, publié par un auteur anonyme vers 1552, et le Guzmán de Alfarache (1599) de Matteo Alemán. Depuis, la notion de roman picaresque a été libérée de son enracinement initial dans la tradition littéraire espagnole des $\mathrm{XVI}^{\mathrm{e}}$ et XVII ${ }^{\mathrm{e}}$ siècles, tout en étant, jusqu'à aujourd'hui, appliquée à un certain type de romans européens. On parle parfois, pour montrer la rupture avec la notion traditionnelle espagnole, de "néopicaresque " ${ }^{6}$. On peut néanmoins constater que tous les romans picaresques, quels que soient l'époque ou le pays de leur origine, disposent de certaines caractéristiques que l'on considère comme cruciales pour le genre. En ce qui concerne les propriétés du picaresque, un travail de Claudio Guillén ${ }^{7}$, publié en 1962, reste primordial - une partie importante des publications subséquentes sur le picaresque s'appuient sur lui ${ }^{8}$ - et nous allons en résumer les points principaux.

Le roman picaresque est caractérisé par des formes de narration autobiographique, assumées en général par un individu marginal par rapport à la société dans laquelle il vit. Ce narrateur homodiégétique et intradiégétique raconte l'histoire de sa vie, ou du moins de certains épisodes de cette vie, qui forment dans l'ensemble une image critique de la société qui entoure le picaro. Celui-ci n'est pas un révolutionnaire; au contraire, pour vivre sa vie d'exclu, de vagabond, de hors-la-loi, il a besoin de cette société - il n'a donc nullement intérêt à changer un système qui, tout en le marginalisant, lui procure des moyens pour survivre. L'attitude du picaro envers la société reste donc ambiguë, du fait de sa propre situation paradoxale face à elle. Ambiguë est aussi la perspective du narrateur, qui d'un côté est fier de raconter ses aventures et les présente en conséquence de manière positive, alors qu'il est, de l'autre côté, un narrateur dont la disposition morale douteuse peut sûrement mener à mettre en cause ce qu'il raconte, ce qui conduit à soupçonner qu'il s'agit d'un narrateur qui ne dit pas la vérité. La position problématique du picaro par rapport à sa propre histoire est encore aggravée par le mode de narration épisodique, dominant dans le roman picaresque, et qui rend

6. Cf. U. Broich, «Der neopikareske Roman».

7. C. Guillén, «Zur Frage der Begriffsbestimmung des Pikaresken».

8. Cf. p. ex. M. Bauer, Der Schelmenroman; H. G. Rötzer, Der europäische Schelmenroman; D. Souiller, Le roman picaresque. 
la narration encore moins fiable aux yeux du lecteur. Le fait qu'incontestablement, le picaro ne présente qu'un choix de ses aventures, implique en effet qu'il y a une partie de sa vie qu'il cache au lecteur. Le caractère fragmentaire du roman picaresque se voit au fait que les épisodes du roman ne sont en général pas reliés entre eux. Si un lien existe entre les épisodes, il est souvent établi après coup et de manière très artificielle par le picaro-narrateur lui-même, mettant ainsi encore plus l'accent sur le caractère fragmentaire de la narration. Le choix des épisodes dépend de l'objectif que le picaro poursuit avec sa narration: veut-il justifier son mode de vie, ce qu'il est devenu, un choix qu'il a pu faire? On peut souvent observer, selon Rötzer, une tension entre le moi narré et le moi narrant $^{9}$ : par rapport à ses actes passés, le picaro peut adopter une attitude distanciée, voire péjorative.

Pour ce qui est des motifs picaresques, je ne mentionnerai que les plus fréquents, que l'on retrouve tous d'une façon ou d'une autre dans le Manuscrit. Didier Souiller les résume en commençant par l'enfance du picaro, qui serait marquée par sa "Naissance et [l']influence du milieu» et par une "Education négligée et [des] mauvais traitements". Le roman picaresque contient «deux expériences clés: la première duperie dont est victime le personnage [...]; la première expérience du vol, fruit du hasard et de la facilité» ${ }^{10}$. Le récit est par la suite organisé par trois principes différents à la fois : "L'itinéraire géographique» que prend le picaro, son "passage par différents maîtres» et "les récits librement insérés » ${ }^{11}$ dans son histoire. Le personnage principal est en général soumis à deux épreuves: il sera mis en prison et il devra subir un amour malheureux ${ }^{12}$. De ces motifs résultent, selon Souiller, plusieurs sujets récurrents: l'argent, le voyage et l'errance, la fortune, l'apparence et l'illusion, la faim, ou plutôt l'expérience de la nécessité matérielle ${ }^{13}$. Tous ces éléments de contenu se retrouvent sous une forme ou sous une autre dans le Manuscrit trouvé à Saragosse.

9. H. G. Rötzer, Der europäische Schelmenroman, p. 120.

Io. D. Souiller, Le roman picaresque, p. 58.

II. Ibid.

I2. Ibid., p. 60.

13. Ibid., p. 60 sq. 
Le picaro comme narrateur - un unreliable narrator?

Comme on l'a vu, le picaro est un narrateur particulier: il raconte sa propre histoire mais il ne la raconte pas, comme Rousseau l'annonce dans ses Confessions, pour se montrer «tel que je fus». Le picaro, avec la distance de l'âge, veut montrer autre chose: il veut dévoiler le fonctionnement d'une société injuste mais il veut aussi montrer sa propre intelligence, sa supériorité, sa capacité de se débrouiller et de duper les autres. Pour y parvenir, le narrateur picaresque utilise, comme le picaro dans sa vie quotidienne, des moyens douteux. Le lecteur se voit rapidement obligé de remettre en cause non seulement les actes du picaro, mais aussi la manière dont il les raconte - il a donc affaire à un imposteur sur deux niveaux. Comme le picaro est, au cours de sa vie, essentiellement entouré de personnages qu'il considère comme des ennemis, la perspective du roman picaresque est fondamentalement partiale; le lecteur ne dispose d'aucune autre version des évènements que celle du picaro lui-même, qui est, très probablement, un menteur.

Dans son travail sur le picaro comme narrateur non fiable, Maren Jäger insiste sur le fait que le roman picaresque établit souvent une relation dialogique entre le narrateur et le lecteur, c'est-à-dire que le narrateur fait de nombreux appels au lecteur pour obtenir sa bienveillance et sa confiance ${ }^{14}$ et obtenir de lui qu'il comble lui-même les vides laissés par la narration. Cette structure dialogique se manifeste dans le Manuscrit par les nombreuses interruptions de la narration lorsque les auditeurs commentent ce que le narrateur leur raconte. A une remarque critique de Rébecca, le bohémien répond:

Madame, répondit le vieux chef, permettez-moi de vous le dire: j'ai cru m'apercevoir que vous vous pressiez trop de porter un jugement sur les récits que j'ai l'honneur de vous faire. [...] Cependant vous en jugerez mieux si vous voulez bien écouter l'histoire de son fils, telle qu'il la raconta au même Cabronez ${ }^{15}$.

Mais le narrateur ne tente pas seulement d'influencer son public, il se leurre aussi lui-même, il essaye de se persuader lui-même de ses propres bonnes intentions, d'argumenter et de se justifier. Cette stratégie de

I4. M. Jäger, «Unzuverlässigkeit im pikarischen Roman», p. 220.

I5. MTS-1810, p. 517. 
persuasion ne passe évidemment pas inaperçue chez le lecteur. C'est surtout du Lazarillo que Jäger parle ici, le Guzman en revanche représenterait plutôt un exemple négatif pour le lecteur: le narrateur se distancie de ses infamies d'autrefois, il rapporte une expérience de conversion ${ }^{16}$. Cette variante du roman picaresque poursuit de toute évidence le but d'une leçon morale pour ses lecteurs qui, à travers le mauvais exemple du picaro, seraient incités à mener une vie plus chrétienne que le héros du roman. C'est d'ailleurs surtout dans cette forme du roman picaresque qu'on peut observer la tension entre le moi narré et le moi narrant mentionnée plus haut; Jäger parle même d'une "[...] Schizophrenie zwischen erzählendem und erlebendem Ich [...] ${ }^{17}$. Mais est-ce que cette mise à distance peut être prise au sérieux par le lecteur? Il semble, selon Jäger, que le narrateur moralisant reste - même lorsqu'il porte un jugement sévère sur soi - peu fiable et que le lecteur doive continuer à le mettre en question:

Wird das Bekehrungserlebnis nicht als überzeugend bewertet, schließt der Leser darauf, dass er es mit einem unzuverlässigen Erzähler zu tun hat, dessen moralische Kommentare scheinheilig und halbherzig sind ${ }^{18}$.

Le jugement du lecteur sur la fiabilité du narrateur picaresque dépend de la qualité de son récit, donc de ses qualités de narrateur. Il faut que le narrateur picaresque arrive à persuader son public de la sincérité de ses remords pour être pris au sérieux par ses lecteurs.

Un troisième aspect du roman picaresque mérite de retenir l'attention dans le contexte du Manuscrit: il y a des textes dans lesquels le problème de l'impossibilité de faire confiance au narrateur se déplace du niveau de la narration à celui du discours, comme dans l'exemple du Tristram Shandy de Sterne ${ }^{19}$. Le narrateur de Sterne parle ouvertement des problèmes qu'il a pour saisir tous les détails dont est composée l'histoire de sa vie et il finit par avouer l'impossibilité d'en contrôler le matériau. Ce dont Tristram se rend compte, c'est qu'on ne peut pas noter tous les détails d'une vie, même pas tout ce qui semble important pour son

I6. Cf. M. Jäger, «Unzuverlässigkeit im pikarischen Roman», p. 222.

I7. Ibid.

I8. Ibid.

19. Ibid., p. 228. 
protagoniste. Selon Jäger, ce texte serait une épopée sur les impossibilités du roman. A partir de ce moment de l'histoire de la littérature, le côté métapoétique sera à tout jamais présent dans les narrations picaresques. Ce nouvel aspect du genre picaresque, omniprésent au XVIII ${ }^{\mathrm{e}}$ siècle, sera, on le verra plus loin, un aspect fécond pour l'analyse du Manuscrit.

\section{Exemple du picaro dans le Manuscrit trouvé à Saragosse}

Comme Hervier le fait remarquer à juste titre, le côté picaresque du personnage d'Avadoro avait déjà été mis en évidence par Caillois luimême ${ }^{20}$. Le bohémien, qui apparaît tantôt sous le nom de Pandesowna, d'Avadoro ou de Don Juan, figure dans le roman de trois façons différentes: il est l'acteur principal de ses propres aventures, il en est le narrateur et il est le narrateur d'un nombre important d'autres histoires dans lesquelles il n'apparaît pas ou très peu. Dans la version de 1810, le chef bohémien est l'unique narrateur de la quinzième à la quarantième journée; dans la version de 1804 , son récit est régulièrement interrompu par celui du Juif errant, mais du point de vue quantitatif il reste de loin - mis à part Alphonse - le narrateur le plus important dans les deux versions du roman.

Jeux de travestis

Comme la plupart des personnages figurant dans le Manuscrit, le chef bohémien se révèle ne pas être celui qu'il prétend. Pourtant, lorsqu'Alphonse le voit pour la première fois, depuis la terrasse du château d'Uzéda ${ }^{21}$, le jeune voyageur n'hésite pas une seconde à l'identifier de loin, à partir de ses vêtements et des circonstances de son apparition, pour ce qu'il semble être: un bohémien. Mais déjà ses deux filles, qu'Alphonse rencontre avant de faire la connaissance de leur père, semblent, d'après les observations du jeune cavalier, jouer un rôle ${ }^{22}$. Lorsqu'elles lui présentent leur père, c'est Alphonse qui précise:

20. Cf. R. Caillois, "Destin d'un homme et d'un livre», p. 41 : "Avadoro est plus picaresque que surnaturel.»

2I. Cf. $M T S-1810$, p. 192.

22. MTS-1810, p. 215. 
[...] elles me présentèrent à un vieillard de bonne mine et encore frais qu'elles me dirent être leur père ${ }^{23}$.

La tournure de la phrase exprime le doute d'Alphonse sur l'identité du vieillard, qui est pourvu d'ailleurs, dès cette première rencontre, "d'un air un peu malin " ${ }^{24}$. Invité à suivre la troupe pendant quelques jours dans son périple dans la Sierra Morena, Alphonse consent sans hésiter à partager désormais leur vie de contrebandiers. Dès le début de son histoire, que le bohémien raconte pour apaiser la curiosité du jeune voyageur ${ }^{25}$, Avadoro révèle qu'il n'est point né bohémien, mais qu'il est fils d'un gentilhomme madrilène. Il raconte à Alphonse des épisodes de sa vie, fragments qui sont censés expliquer comment le fils d'un gentilhomme de Madrid a fini par mener une vie de hors-la-loi dans les montagnes de la Sierra Morena. Au cours de ces épisodes, toujours interrompus par d'autres histoires tout aussi fragmentaires que celle du bohémien, Avadoro, comme un picaro typique, modifie à plusieurs reprises son apparence physique, car:

[...] le picaro se déguise constamment pour chercher à s'identifier à son habit $[\ldots]^{26}$.

Avadoro cache sa véritable identité, il change d'habits, de profession voire de sexe pour des raisons variées, qu'il se refuse à révéler à son auditeur. Il semble qu'il n'y ait aucune nécessité visible dans la succession des événements d'une autobiographie souvent à la limite du vraisemblable; un épisode ne mène pas logiquement au prochain, ni dans la chronologie de sa vie, ni dans celle du texte. Lorsque le jeune Avadoro, après une suite d'aventures bien picaresques, impliquant déjà des déguisements multiples, s'enfuit de la maison de la duchesse de Sidonia, il décide de s'habiller en mendiant:

[...] réfléchissant sur le meilleur parti que j’avais à prendre, il me parut que c'était d'embrasser l'état de mendiant pour tout le temps que devait durer ma pénitence ${ }^{27}$.

23. MTS-1810, p. 215.

24. MTS-1810, p. 215.

25. MTS-1810, p. 219.

26. D. Souiller, Le roman picaresque, p. 64.

27. MTS-1810, p. 396. 
Le changement d'identité s'effectue par une transformation vestimentaire et même une sorte de masque:

Je vis ma figure dans une glace et je trouvai que mon extérieur ne répondait pas assez à l'état que j'allais embrasser. Je pris un charbon dans une brasière et m'en servis à modérer l'éclat de mon teint, ensuite je déchirai ma chemise et mon habit $[\ldots]^{28}$.

Le déguisement de mendiant permet au narrateur de faire changer son personnage de lieu et de patron en même temps, comme le picaro a coutume de le faire. Le patron du picaro s'incarne dans le Manuscrit en une série de personnages auxquels Avadoro est confronté au cours de sa vie - il ne s'agit pas forcément d'un patron dans le sens propre du mot, ce peut être des compagnons de voyage ${ }^{29}$, des professeurs ${ }^{30}$ ou des gardiens de prison ${ }^{31}$. La fonction du personnage du "patron" est double: il pousse le jeune picaro à faire quelque chose, donc à vivre l'aventure qu'il va raconter à ses lecteurs et en même temps, il lui raconte sa propre vie, ce qui lui permet donc de présenter aux lecteurs un récit encadré. Dans son déguisement de mendiant, le bohémien travaille d'abord pour le Chevalier de Tolède, un libertin qu'il assiste pour accomplir ses aventures amoureuses. Au cours d'une péripétie invraisemblable, Avadoro échange, après avoir passé un certain temps chez le Chevalier, son poste avec un "collègue» qui, lui, travaille pour un jeune négociant, Lope Soarez:

[...] je le priais de prendre ma place auprès du chevalier seulement pour quelques jours. Il me répondit qu'il le ferait volontiers [... ${ }^{32}$.

Cette manœuvre artificielle permet au narrateur d'introduire le personnage du négociant dont il raconte l'histoire, et de faire vivre à son personnage picaresque une autre aventure aux côtés de ce jeune bourgeois originaire de Cadix qui, chose étrange, consent sans aucune hésitation, comme le Chevalier, à l'échange de son valet:

28. MTS-1810, p. 397.

29. Cf. l'épisode avec le vice-roi du Mexique.

30. Cf. l'épisode avec le père Sanudo.

3I. Cf. l'épisode avec la Duchesse de Sidonia.

32. MTS-1810, p. 410. 
Je dis que j'étais venu à la place de mon petit camarade Chiquito, que je m'appelais Avarito, enfin que je rendais les mêmes services et avec la même fidélité. On me répondit que mes services seraient acceptés ${ }^{33}$.

La manière manifestement forcée dont le bohémien enchaîne les événements de sa vie au cours de sa narration peut mener à l'hypothèse que ce qu'il veut raconter à ses auditeurs, ce n'est pas tant sa propre vie, mais plutôt une succession d'épisodes exemplaires de la vie d'un picaro. L'existence d'Avadoro ne correspond pas véritablement au modèle picaresque, car il ne souffre jamais ni de faim, ni de froid, ni d'injustice; en outre, étant d'origine noble il ne peut pas être un authentique picaro. Néanmoins, en racontant des épisodes à caractère évidemment picaresque, il essaye bel et bien de se faire passer pour tel. Aussi le récit de sa vie ne peut-il pas être, comme tout récit biographique, téléologique (ni la fin du récit, ni celle du Manuscrit ne donnent au lecteur une image définitive de l'identité du bohémien au moment où il raconte sa vie), car il est soumis à la volonté d'intégrer dans la narration des lieux communs du genre picaresque. Le vrai picaro est livré au bon plaisir de Fortuna ${ }^{34}$, comme le lecteur du Manuscrit est livré aux humeurs de ce faux narrateur picaresque qu'est Avadoro, qui présente dans son roman une accumulation de stéréotypes picaresques, tout en gardant une certaine distance ironique par rapport à ce genre littéraire.

\section{Avadoro comme narrateur picaresque}

Le lecteur sait qu'Avadoro en tant que personnage picaresque est capable de mentir et le fait d'ailleurs souvent - par exemple quand il fait semblant d'être un mendiant pour échapper à une punition ou quand il se fait passer pour une fille pour permettre à celle-ci de s'enfuir avec son amant. Evidemment, le lecteur se doute qu'Avadoro est aussi capable de mentir en tant que narrateur picaresque. Les travestissements divers du bohémien reflètent effectivement son attitude de narrateur: il n'arrête pas de jouer des tours aux lecteurs, en sautant d'une histoire à l'autre et en présentant parfois des perspectives différentes sur un même incident. Avadoro - comme d'ailleurs la plupart des narrateurs dans le Manuscrit-

33. MTS-1810, p. 411.

34. Comme le dit d'ailleurs le narrateur du Lazarillo dans sa préface. 
est un narrateur non fiable, il ne donne pas toutes les informations dont il dispose ou bien il met volontairement son public sur une fausse piste.

Dans l'épisode déjà cité, parlant du Chevalier de Tolède et du négociant Lope Soarez, Avadoro dit avoir entendu une voix que son patron prenait pour celle de son ami mort, le Chevalier d'Aguilar:

Ah! mon ami, dit le chevalier, quand une fois l'on entend la voix des morts, on n'a pas longtemps à rester parmi les vivants ${ }^{35}$.

Un peu plus tard, le même épisode est raconté par Lope Soarez, qui dit être monté à une fenêtre derrière laquelle il espérait trouver son amante; Avadoro interrompt le récit du négociant:

Ah! m'écriais-je, pauvre Seigneur don Lope, il n'en faut pas douter: la maison où vous êtes monté était celle du chevalier de Tolède. Nous y attendions l'âme du Chevalier d'Aguilar qui venait d'être tué en duel. Quand vous avez frappé contre le volet, nous vous avons pris pour un habitant d'un autre monde, et voilà pourquoi nous vous avons demandé des nouvelles du purgatoire... ${ }^{36}$

Bien qu'Avadoro en tant que narrateur connaisse, au moment où il raconte la première version de l'épisode, l'explication donnée dans la version du négociant, il préfère laisser ses auditeurs dans l'ignorance pendant un certain temps.

Par rapport aux trois différents aspects du narrateur picaresque introduits plus haut, Avadoro représente certes un mélange intéressant: le but de sa narration est, d'un point de vue picaresque, incertain. L'histoire du bohémien ne peut être lue comme un épisode qui servirait à expliciter une critique de la société: Avadoro est de naissance noble; malgré ses aventures diverses, il ne souffre jamais et s'il perd son amour, c'est par la faute des intrigues d'un personnage qui est encore plus rusé, plus picaresque en quelque sorte, que lui, Busqueros. Il y a, certes, des passages qui laissent supposer qu'il s'agit bien de l'histoire de la conversion d'un picaro:

Mais en vérité, dit le Bohémien en s'interrompant lui-même, ce n'est pas sans quelque peine que je vous parle de jeux aussi coupables. Notre 
extrême jeunesse pouvait seule les excuser, et si je ne comptais sur votre indulgence, je n'oserais jamais poursuivre mon récit ${ }^{37}$.

Mais cette interruption du bohémien est supprimée dans la version de 1810, et le fait qu'Avadoro finisse visiblement par devenir un criminel, un contrebandier hors-la-loi, devrait être suffisant pour prouver qu'il ne regrette pas vraiment ses actes antérieurs, qu'il ne s'agit donc pas de l'histoire d'une conversion.

Reste la question de l'aspect métapoétique, qui est beaucoup plus présente dans la version de 1804, comme le prouve un exemple:

En vérité, je redoute extrêmement cette histoire; toutes celles du Bohémien commencent d'un air fort simple et l'on espère en voir bientôt la fin: point du tout, une histoire en referme une autre qui en contient une troisième. [...] Au lieu que si je veux prendre la somme de tout ce que dit le Bohémien, je n'y trouve rien qu'une extrême confusion ${ }^{38}$.

Ce n'est pas le narrateur même qui met en question le discours narratif, mais ce sont ses auditeurs, comme le géomètre Velasquez, qui le fera, même dans la version de 1810, à plusieurs reprises:

J'avais bien prévu que les histoires du Bohémien s'engraineraient [sic] les unes dans les autres. [...] s'il met encore une histoire à la traverse je me brouillerai avec lui $[\ldots]^{39}$.

Rébecca se plaint plusieurs fois des interruptions dans la narration du bohémien: «En vérité, je suis très fâchée de cette interruption $[\ldots]$ » 40 , dit-elle; un peu plus loin Alphonse note:

Rébecca observa avec une sorte d'impatience que nous étions toujours interrompus à l'endroit d'une histoire le plus intéressant ${ }^{41}$.

La manière dont le bohémien présente son histoire est le sujet de diverses discussions entre les voyageurs, donnant effectivement une dimension

37. MTS-1804, p. 431 sq.

38. MTS-1804, p. 498 sq.

39. MTS-1804, p. 588 sq.

40. MTS-1810, p. 282.

4I. $M T S-1810$, p. 305. 
métanarrative au roman. On lui reproche de jouer des tours à ses auditeurs en mettant volontairement leur patience à l'épreuve et en faisant exprès de les déconcerter. Au cours de la trente-huitième journée, le bohémien s'interrompt encore:

Ici finit l'histoire de mon enfance. Je vous l'ai dite avec de grands détails parce que toutes les circonstances en sont encore présentes à ma mémoire. [...] Je ne puis vous promettre la même exactitude pour l'histoire de ma jeunesse. Si je reporte mon imagination à cette brillante époque de ma vie, je n'y distingue qu'un tumulte de passions diverses et comme le trouble confus des orages. Un entier oubli me dérobe des choses qui alors remplissaient mon âme et les plaisirs qui la ravissaient ${ }^{42}$.

Cependant, le bohémien continue à raconter l'histoire de sa jeunesse, tout en ayant averti le public qu'il a oublié une grande partie de ce qu'il veut raconter et en faisant, de façon explicite, référence à l'imagination qui semble se substituer à la mémoire pour produire le récit autobiographique. Les difficultés de représenter toute une vie dans un roman, décrites par Jäger, sont donc présentes dans le discours du bohémien et elles le sont de manière ouverte. C'est donc surtout l'aspect métapoétique du narrateur picaresque que l'on retrouve chez le bohémien Avadoro.

\section{Conclusion}

Il est certain que plusieurs raisons permettent de conclure que le Manuscrit trouvé à Saragosse n'est pas un roman picaresque. Son personnage principal, le jeune Adolphe, n'est pas un picaro et le roman manque de la dimension critique qui caractérise en général le roman picaresque. Néanmoins, le personnage majeur du bohémien Avadoro semble vouloir se faire passer pour un personnage picaresque. Pour ceci, il se sert d'un côté d'un choix d'épisodes picaresques, c'est-à-dire de fragments stéréotypés au niveau du contenu, de topoï qui fonctionnent comme des citations du genre et, d'un autre côté, il développe, en tant que narrateur, cette attitude si caractéristique des narrateurs picaresques qui passent leur temps à tromper leurs auditeurs ou leurs lecteurs.

42. MTS-1810, p. 571. 
Même si la présence du picaresque n'est pas décelable dans tout le roman, même si elle se limite à des citations d'épisodes stéréotypes, des topoï, même s'il ne s'agit pas d'un picaresque authentique, mais d'un faux picaresque, il sert quand même à attirer l'attention du lecteur sur le mensonge comme principe narratif. Le mensonge est de fait un principe narratif omniprésent dans le Manuscrit: le complot de la société secrète des Gomelez, élucidé à la fin du roman, consiste dans une énorme escroquerie, mise en scène par ses membres pour mettre Alphonse à l'épreuve. L'ermite chrétien qu'Alphonse rencontre lors de sa seconde journée dans la Sierra Morena se révèle être le chef des Gomelez:

Jeune Nazaréen, me dit le scheik, vous reconnaissez en moi l'ermite qui vous reçut dans la vallée du Guadalquivir, mais vous devinez assez que je suis le grand scheik des Gomelez ${ }^{43}$.

Le scheik avoue que les Gomelez avaient «arrangé toutes les circonstances de [l'] arrivé» d'Alphonse, pour le «rendre musulman» ou au moins le "rendre père " ${ }^{44}$. Le "terrible démoniaque Pascheco " n'est "qu'un saltimbanque basque» "45 dont l'histoire fictive était censée impressionner Alphonse et l'entraîner à trahir son honneur. Or, tandis que l'identité des autres personnages qui font partie du complot n'est révélée qu’à la fin du roman, le bohémien, personnage pseudo-picaresque, ne cache nullement son identité multiple, et ce dès le début.

Mais la question qui se pose n'est pas de savoir si l'histoire racontée correspond à la vérité ou si la personne qui la raconte l'a vraiment vécue, mais de savoir s'il s'agit d'une bonne histoire, d'une histoire qui produit un effet sur son auditeur. La réponse d'Avadoro, lorsqu'Alphonse lui dit que l'histoire de Giulio Romati, que le bohémien vient de présenter à ses auditeurs comme une histoire vraie, figure aussi dans un livre de Hapélius, correspond à cette hypothèse:

Cela peut être, reprit le chef. Peut-être Romati a-t-il pris son histoire dans un livre, peut-être l'a-t-il inventée. Toujours est-il sûr que son récit contribua beaucoup à me donner le goût des voyages et même un 
espoir vague de trouver des aventures merveilleuses que je ne trouvai jamais ${ }^{46}$.

Ce n'est pas l'originalité d'une histoire qui justifie qu'on la raconte, mais la réaction qu'elle provoque chez le public, et ceci certainement à travers la qualité du récit. L'insertion du narrateur picaresque - et du mensonge comme principe de la narration - dans le Manuscrit trouvé à Saragosse attire l'attention du lecteur sur le fait que, bien que l'on ne puisse jamais faire confiance au narrateur, que l'on puisse toujours remettre en question ce qui est raconté, cela ne change rien au pouvoir du narrateur de donner à l'existence de ceux qui l'écoutent une orientation nouvelle, comme l'histoire de Romati donne l'envie des aventures à Avadoro.

\author{
Lena Seauve \\ Humboldt-Universität zu Berlin
}

46. MTS-1810, p. 252. 


\section{BIBLIOGRAPHIE}

Textes

MTS-1804 et MTS-1810 = Ротоскі, Jean, Manuscrit trouvé à Saragosse (version de 1804 et version de 1810), éd. par François Rosset, Dominique Triaire, Paris, Flammarion, 2008 (GF 1342-1343).

\section{Etudes}

Bauer, Matthias, Der Schelmenroman, Stuttgart, J. B. Metzler, 1994.

BRoICH, Ulrich, "Der neopikareske Roman", in Gattungen des modernen englischen Romans, hrsg. von Ulrich Broich, Wiesbaden, Athenaion, 1975, p. 143-175.

Caillois, Roger, "Destin d'un homme et d'un livre: Le comte Jean Potocki et Manuscrit trouvé à Saragosse", in Manuscrit trouvé à Saragosse, éd. par Roger Caillois, Paris, Gallimard, 1958, p. 9-45.

Guillén, Claudio, "Zur Frage der Begriffsbestimmung des Pikaresken", in Pikarische Welt: Schriften zum europäischen Schelmenroman, hrsg. von Helmut Heidenreich, Darmstadt, Wiss. Buchges., 1969. Hervier, Julien, "Jean Potocki ou les métamorphoses du picaresque", in De Shakespeare à Michel Butor. Mélanges offerts à Monsieur Charles Dédéyan, Paris, Presses de l'Université de Paris Sorbonne, 1985, p. 43-53.

JÄGER, Maren, "Unzuverlässigkeit im pikarischen Roman" in Was stimmt denn jetzt? Unzuverlässiges Erzählen in Literatur und Film, hrsg. von Fabienne Liptay, Yvonne Wolf, München, Text und Kritik, 2005, p. 218-232.

Rosset, François, «Le langage du fantastique. Stratégies et fatalité du réemploi», Poétique, 166 (2011), p. 203-214.

Rötzer, Hans Gerd, Der europäische Schelmenroman, Stuttgart, Reclam, 2009. 
Souiller, Didier, Le roman picaresque, Paris, Presses universitaires de France, 1989.

Zurowski, Maciej, «Le Manuscrit trouvé à Saragosse et la technique romanesque du XVIII e siècle", in Jean Potocki et le manuscrit trouvé à Saragosse, éd. par Elzbieta Grabska-Wallis, Warszwa, 1972, p. 105-112. 